\title{
CP-violation studies of hyperon-antihyperon pairs with BESIII
}

\author{
P. Adlarson ${ }^{a, *}$ and K. Schönning, for the BESIII collaboration ${ }^{a}$ \\ ${ }^{a}$ Department of Physics and Astronomy, \\ Box 516, SE-751 20, Uppsala, Sweden \\ E-mail: patrik.adlarson@physics.uu.se, karin.schonning@physics.uu.se
}

The BESIII experiment at the electron-positron collider BEPCII in Beijing has collected the world's largest data at the $J / \psi$ and $\psi(2 S)$ resonances. The fact that the hyperons are produced with a non-zero spin polarization that is straightforward to parameterize in processes involving virtual photons or vector mesons, enable direct and precise CP tests. These CP-symmetry tests can be performed on e.g. $e^{+} e^{-} \rightarrow J / \psi \rightarrow \Lambda \bar{\Lambda} \rightarrow p \pi^{-} \bar{p} \pi^{+}, e^{+} e^{-} \rightarrow J / \psi \rightarrow \Sigma^{+} \bar{\Sigma}^{-} \rightarrow p \pi^{0} \bar{p} \pi^{0}$ or $e^{+} e^{-} \rightarrow J / \psi \rightarrow \Xi^{-} \bar{\Xi}^{+} \rightarrow \Lambda \pi^{-} \bar{\Lambda} \pi^{+} \rightarrow p \pi^{-} \pi^{-} \bar{p} \pi^{+} \pi^{+}$. For the $\Xi^{-} \rightarrow \Lambda \pi^{-}$decay the exclusive measurement of the final state particles allows for three independent CP tests and the determination of the strong and weak phase differences. The weak phase difference is the first measurement for any baryon decay. The decay parameter $\phi_{\Xi}$ is measured with a precision similar to the hitherto most precise measurement, performed by the HyperCP experiment at Fermilab. This is despite the fact that the BESIII data sample is three orders of magnitude smaller than that of HyperCP.

\footnotetext{
*** Particles and Nuclei International Conference - PANIC2021 ***

*** 5 - 10 September, $2021 * * *$

*** Online ***
}

\footnotetext{
${ }^{*}$ Speaker
} 


\section{Introduction}

That the combined charge and parity symmetry $(\mathrm{CP})$ is violated in weak decay processes has been known for more than four decades, yet there are still many aspects left to explore. The overarching question is what role this fundamental symmetry breaking plays in baryogenesis. In the quark sector, the existence of CP violation in kaon and beauty meson decays is well established [1-3]. Only recently the first CP-violating signal in a charmed meson system was measured [4]. Up until now all confirmed instances have been observed in the meson sector. An important milestone would be to discover CP-violation in baryonic weak decays. In recent years the BESIII experiment has performed several proof-of-concept measurements, showing the potential of testing $\mathrm{CP}$ in strange baryon weak decays. This contribution aims to give a short overview of this progress.

\section{Direct $\mathbf{C P}$ violation in strange decays}

The direct $\Delta S=1 \mathrm{CP}$ violating contribution is parametrized by the complex parameter $\epsilon^{\prime}$. It is related to the corresponding indirect contribution $\epsilon$ via $\operatorname{Re}\left(\epsilon^{\prime} / \epsilon\right)$. This value has been determined by the KTeV [5, 6] and NA48 [7] experiments from two-pion decay rates of neutral kaons and the combined result, $\operatorname{Re}\left(\epsilon^{\prime} / \epsilon\right)=(16 \pm 2.3) \times 10^{-4}$, is consistent with the Standard Model prediction. The theory prediction for $\operatorname{Re}\left(\epsilon^{\prime} / \epsilon\right)$ has however been challenging to compute as its value comes from partially canceling contributions of electroweak and QCD penguin diagrams, see Ref. [8] and references therein. Only recently different theoretical approaches have found consensus for the electroweak part [9-11], while the disagreement for the QCD contribution remain [12]. Hyperon weak decays offer a complementary approach of testing $\Delta S=1 \mathrm{CP}$ violation compared to $K \rightarrow$ $\pi \pi[13]$.

\section{Hyperon decays}

A hyperon $Y$ which decays into a spin $1 / 2$ baryon $B$ and a pion $\pi$, e.g. $\Lambda \rightarrow p \pi^{-}$, consists of a parity violating $(S)$ and a parity conserving $(P)$ amplitude. These can be written as $S=|S| e^{i \delta_{S}} e^{i \xi_{S}}$ and $P=|P| e^{i \delta_{P}} e^{i \xi_{P}}$, where $\xi$ and $\delta$ are the weak and strong phases, respectively. The full angular decay distribution is described with three decay parameters $\alpha$ and $\beta$ and $\gamma$ which parametrizes the $S$ and $P$ wave intereference. Experimentally, a more natural choice is to determine the two uncorrelated parameters $\alpha$ and $\phi=\tan ^{-1}(\beta / \gamma)$. The $\alpha$ parameter can be extracted in all singly weak decays if the mother hyperon $(\Lambda$ and $\Sigma$ ) is polarized, while the $\phi$ parameter can only be measured in sequential weak decays like $\Xi \rightarrow \Lambda \pi \rightarrow p \pi \pi$. By measuring the corresponding parameters $\bar{\alpha}$ and $\bar{\phi}$ for the antihyperon, two CP-tests can be constructed. In the CP-conserving limit $\alpha=-\bar{\alpha}$ and $\phi=-\bar{\phi}$, and therefore one can construct

$$
A_{\mathrm{CP}}=\frac{\alpha+\bar{\alpha}}{\alpha-\bar{\alpha}} \approx-\sin \phi \frac{\sqrt{1-\alpha^{2}}}{\alpha}\left(\xi_{P}-\xi_{S}\right), \Delta \phi_{\mathrm{CP}}=\frac{\phi+\bar{\phi}}{2} \approx \cos \phi \frac{\alpha}{\sqrt{1-\alpha^{2}}}\left(\xi_{P}-\xi_{S}\right),
$$

where $\left(\xi_{P}-\xi_{S}\right)$ is the CP-sensitive weak phase difference. As $\phi$ is small, $\Delta \phi_{\mathrm{CP}}$ is an order of magnitude more sensitive compared to $A_{\mathrm{CP}}$ [14]. The CP-violating contribution for $A_{\mathrm{CP}}$ and $\Delta \phi_{\mathrm{CP}}$ assuming the Standard Model is predicted to be $O\left(10^{-5}\right)$ and $O\left(10^{-4}\right)$, respectively [15]. Up until 
now no test on $\Delta \phi_{\mathrm{CP}}$ has been performed. Instead, the most precise test was performed by the HyperCP collaboration on the sum $A_{\mathrm{CP}}^{\Lambda}+A_{\mathrm{CP}}^{\Xi}=\left(0.0 \pm 5.5_{\text {stat }} \pm 4.4_{\text {syst }}\right) \times 10^{-4}[16]$.

\section{Production of hyperon-antihyperon pairs at $e^{+} e^{-}$colliders}

At the BESIII experiment $Y$ and $\bar{Y}$ are produced in the process $e^{+} e^{-} \rightarrow J / \psi \rightarrow Y \bar{Y}$. The production process is described by two helicity amplitudes denoted $G_{E}^{\Psi}$ and $G_{M}^{\Psi}$, respectively, see Ref. [17] and references therein. The amplitudes are parametrized by two real parameters, $\alpha_{\psi}$, describing the angular distribution of the production process, and $\Delta \Phi=\arg \left(G_{E}^{\Psi} / G_{M}^{\Psi}\right)$. For single weak decays a simultaneous measurement of $\alpha$ and $\bar{\alpha}$ is only possible if the hyperons are polarized, which is equivalent of having $\Delta \Phi \neq 0$. The formalism which describes the production and subsequent decay process of the hyperon-antihyperon pairs as well as the experimentally measured observables are described in detail in Ref. [18]. The complete angular distribution for the single $(a)$ and sequential (b) weak decay chains are given by

$$
\boldsymbol{W}(\xi ; \omega)=\sum_{\mu, v=0}^{3} C_{\mu \nu} D_{\mu \nu}^{Y} ; \quad \text { (a) } D_{\mu \nu}^{\Lambda / \Sigma}=a_{\mu 0}^{\Lambda / \Sigma} a_{\nu 0}^{\bar{\Lambda} / \bar{\Sigma}}, \quad \text { (b) } D_{\mu \nu}^{\Xi}=a_{\mu \mu^{\prime}}^{\Xi} a_{\nu \nu^{\prime}}^{\bar{\Xi}} a_{\mu^{\prime} 0}^{\Lambda} a_{\nu^{\prime} 0}^{\bar{\Lambda}} .
$$

The measured variables in $\xi$ are the spherical coordinates in the helicity frames. There are five and nine such helicity angles for the single and sequential weak decays, respectively. The vector $\omega$ contains the production and decay parameters. The production process is described by the $4 \times 4$ spin density matrix $C_{\mu \nu}$ and the decay chains are given by the $4 \times 4$ decay matrices $a_{\mu \nu}^{Y}$ [18].

\section{Experimental procedure}

The results come from data collected with the BESIII magnetic spectrometer located in Beijing, China [19]. The multi-purpose experimental setup has collected the world's largest charmonium data sample, and the published results are based on $1.3 \times 10^{9} \mathrm{~J} / \psi$ events. For these measurements all final state decay particles are reconstructed. This allows for final event samples with low levels of background contributions. For the $\Lambda \bar{\Lambda}$ and $\Xi^{-} \bar{\Xi}^{+}$it is only a fraction of a percent, while for the $\Sigma^{+} \bar{\Sigma}$ it is approximately $5 \%$. The parameters in $\omega$ are determined by an unbinned maximum log likelihood fit.

\section{Results}

The results are summarized in Table 1. The first polarization measurement for $e^{+} e^{-} \rightarrow J / \psi \rightarrow Y \bar{Y}$ is determined for $J / \psi \rightarrow \Lambda \bar{\Lambda}$ [20]. Here, $\Delta \Phi$ is found to be non-zero and the results translates to a maximum polarization of $25 \%$. The $\Sigma^{+} \bar{\Sigma}^{-}$and $\Xi^{-} \bar{\Xi}^{+}$are also found to be polarized [21, 22]. Therefore, it is possible to simultaneously determine $\alpha$ and $\bar{\alpha}$ and hence also $A_{\mathrm{CP}}$. The measured $A_{\mathrm{CP}}$ are consistent with CP conservation at the level of $O\left(10^{-2}\right)$. Furthermore $\Delta \phi_{\mathrm{CP}}^{\Xi \rightarrow \Lambda \pi}$ is the first CP-test of its kind for any weakly decaying baryon. The weak and strong phase differences are determined to be $\left(\xi_{P}-\xi_{S}\right)_{\Xi \rightarrow \Lambda \pi}=\left(1.2 \pm 3.4_{\text {stat }} \pm 0.8_{\text {syst }}\right) \times 10^{-2} \mathrm{rad}$ and $\left(\delta_{P}-\delta_{S}\right)_{\Xi \rightarrow \Lambda \pi}=$ $\left(-4.0 \pm 3.3_{\text {stat }} \pm 1.7_{\text {syst }}\right) \times 10^{-2}$ rad, respectively. As CP conservation holds at this level of precision, the average values of the decay parameters can also be calculated, $\langle\alpha\rangle=(\alpha-\bar{\alpha}) / 2$, see Table 1 . 
Table 1: Experimentally measured values of the two-body weak decays from BESIII mentioned in these proceedings.

\begin{tabular}{lccccrc}
\hline & $\Delta \Phi($ rad. $)$ & $\langle\alpha\rangle$ & $\langle\phi\rangle(\mathrm{rad})$. & $A_{\mathrm{CP}} \times 10^{3}$ & $\Delta \phi_{\mathrm{CP}}^{\Xi} \times 10^{3}$ (rad.) & Ref. \\
\hline$\Lambda \rightarrow p \pi^{-}$ & $0.740(10)(9)$ & $0.754(3)(2)$ & & $-6(12)(7)$ & & {$[20]$} \\
& & $0.760(6)(3)$ & & $-4(12)(9)$ & {$[21]$} \\
$\Sigma^{+} \rightarrow p \pi^{0}$ & $-0.270(12)(9)$ & $-0.994(4)(2)$ & & $-4(37)(10)$ & & {$[22]$} \\
$\Xi^{-} \rightarrow \Lambda \pi^{-}$ & $1.213(46)(16)$ & $-0.373(5)(2)$ & $0.016(14)(7)$ & $6(13)(6)$ & $-5(14)(3)$ & {$[21]$} \\
\hline
\end{tabular}

For $\alpha_{\Lambda}$ there are now two independent readings from BESIII, both deviating from the recent CLAS determined value, $\alpha_{\Lambda}=0.721 \pm 0.006_{\text {stat }} \pm 0.005_{\text {syst }}$, [20, 21, 23]. The $\phi_{\Xi}$ average differs from the HyperCP measurement, $\phi_{\Xi}=-0.042 \pm 0.011_{\text {stat }} \pm 0.011_{\text {syst }}$ rad, by 2.6 standard deviations [24].

\section{Outlook}

The BESIII experiment has recently collected $10^{10} \mathrm{~J} / \psi$ events which will further reduce the statistical uncertainty of the observables given in Table 1 . There are also other hyperon weak decays currently being studied at BESIII, thus one can expect more results from the collaboration on this topic in the future.

\section{Acknowledgement}

We acknowledge the support of Olle Engkvist Foundation, No. 200-0605 (PA), LundströmÅman Foundation (PA), The Knut and Alice Wallenberg Foundation No. 2016.0157 (PA and KS) and The Swedish Research Council No. 2019-04594 (KS).

\section{References}

[1] J.H. Christenson, J.W. Cronin, V.L. Fitch and R. Turlay, Evidence for the $2 \pi$ Decay of the $K_{2}^{0}$ Meson, Phys. Rev. Lett. 13 (1964) 138.

[2] BABAR collaboration, Observation of CP violation in the $B^{0}$ meson system, Phys. Rev. Lett. 87 (2001) 091801.

[3] Belle collaboration, Observation of large CP violation in the neutral B meson system, Phys. Rev. Lett. 87 (2001) 091802.

[4] LHCв collaboration, Observation of CP Violation in Charm Decays, Phys. Rev. Lett. 122 (2019) 211803.

[5] KTEV collaboration, Measurements of direct CP violation, CPT symmetry, and other parameters in the neutral kaon system, Phys. Rev. D 67 (2003) 012005 [hep-ex/0208007].

[6] KTEV collaboration, Precise Measurements of Direct CP Violation, CPT Symmetry, and Other Parameters in the Neutral Kaon System, Phys. Rev. D 83 (2011) 092001 [1011. 0127]. 
[7] NA48 collaboration, A Precision measurement of direct CP violation in the decay of neutral kaons into two pions, Phys. Lett. B 544 (2002) 97 [hep-ex/0208009].

[8] A. Buras, The $\varepsilon^{\prime} / \varepsilon$-story: 1976-2021, Acta Physica Polonica B 52 (2021) 7.

[9] RBC, UKQCD collaboration, Direct $C P$ violation and the $\Delta I=1 / 2$ rule in $K \rightarrow \pi \pi$ decay from the standard model, Phys. Rev. D 102 (2020) 054509 [2004 . 09440].

[10] A.J. Buras and J.-M. Gérard, Upper bounds on $\varepsilon^{\prime} / \varepsilon$ parameters $B_{6}^{(1 / 2)}$ and $B_{8}^{(3 / 2)}$ from large $N$ QCD and other news, JHEP 12 (2015) 008 [1507.06326].

[11] V. Cirigliano, H. Gisbert, A. Pich and A. Rodríguez-Sánchez, Theoretical status of $\varepsilon^{\prime} / \varepsilon, J$. Phys. Conf. Ser. 1526 (2020) 012011 [1912 . 04736].

[12] J. Aebischer, C. Bobeth, A.J. Buras and J. Kumar, BSM Master Formula for $\varepsilon^{\prime} / \varepsilon$ in the WET Basis at NLO in QCD, 2107.12391.

[13] J. Tandean, New physics and CP violation in hyperon nonleptonic decays, Phys. Rev. D 69 (2004) 076008 [hep-ph/0311036].

[14] J.F. Donoghue, X.-G. He and S. Pakvasa, Hyperon Decays and CP Nonconservation, Phys. Rev. D34 (1986) 833.

[15] J. Tandean and G. Valencia, CP violation in hyperon nonleptonic decays within the standard model, Phys. Rev. D67 (2003) 056001.

[16] HYPERCP collaboration, Search for CP violation in charged-Xi and Lambda hyperon decays, Phys. Rev. Lett. 93 (2004) 262001.

[17] G. Fäldt and A. Kupsc, Hadronic structure functions in the $e^{+} e^{-} \rightarrow \bar{\Lambda} \Lambda$ reaction, Phys. Lett. B 772 (2017) 16 [1702.07288].

[18] E. Perotti, G. Fäldt, A. Kupsc, S. Leupold and J.J. Song, Polarization observables in $e^{+} e^{-}$ annihilation to a baryon-antibaryon pair, Phys. Rev. D99 (2019) 056008.

[19] BESIII collaboration, Design and Construction of the BESIII Detector, Nucl. Instrum. Meth. A 614 (2010) 345 [0911.4960].

[20] BESIII collaboration, Polarization and Entanglement in Baryon-Antibaryon Pair Production in Electron-Positron Annihilation, Nature Phys. 15 (2019) 631.

[21] M. Ablikim et al., Weak phases and CP-symmetry tests in sequential decays of entangled double-strange baryons, May, 2021.

[22] BESIII collaboration, $\Sigma^{+}$and $\bar{\Sigma}^{-}$polarization in the $J / \psi$ and $\psi(3686)$ decays, Phys. Rev. Lett. 125 (2020) 052004 [2004.07701].

[23] D.G. Ireland, M. Döring, D.I. Glazier, J. Haidenbauer, M. Mai, R. Murray-Smith et al., Kaon Photoproduction and the $\Lambda$ Decay Parameter $\alpha_{-}$, Phys. Rev. Lett. 123 (2019) 182301 [1904.07616].

[24] HyPERCP collaboration, New measurement of $\Xi^{-} \rightarrow \Lambda \pi^{-}$, Phys. Rev. Lett. 93 (2004) 011802. 